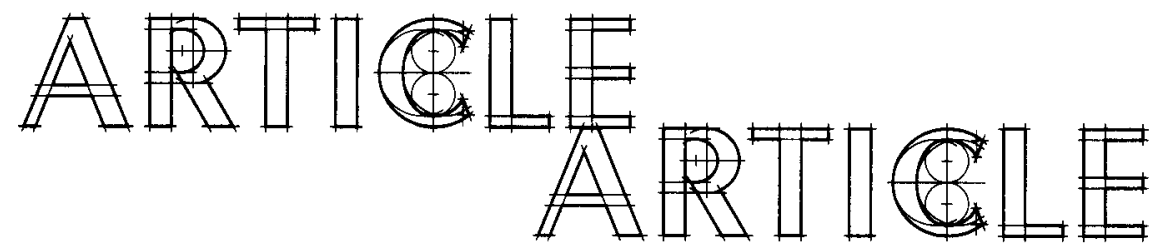

\title{
Complaint of blood running out of eye leads to discovery of elevated choroidal mass
}

\section{Un cas de sang qui s'écoule de l'œil mène à la découverte d'une masse choroïdienne élevée}

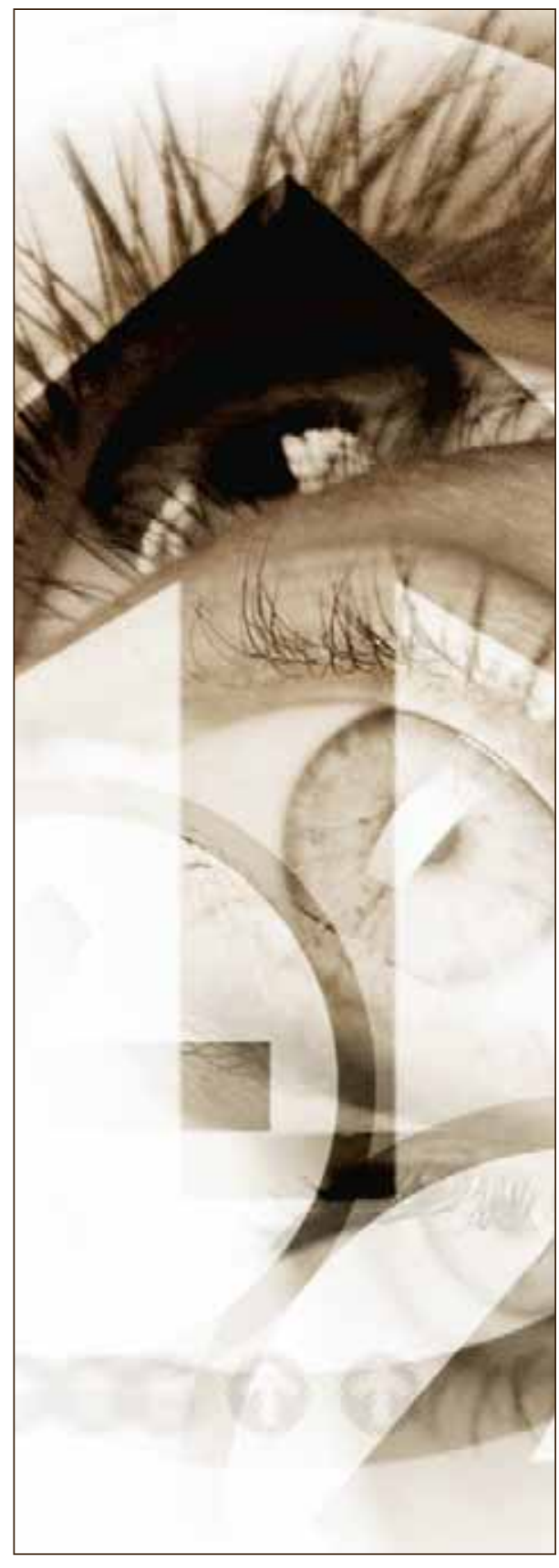

A

27 year old C aucasian female patient presented for examination with symptoms of bleeding from her eye. She had no visual omplaints, though she did note a tenderness to her upper lid when putting pressure on it. $\mathrm{H}$ er health history, as well as her family history, was remark able for hereditary hemorrhagic telangiectasia. She was taking no medications at the time of ex amination.

The patient presented with orrected acuities of $20 / 20$ $0 \mathrm{U}$. H er current refractive error was $-2.25-0.75 \mathrm{x}$ 002 OD and $-3.25-0.50 \times 180$ OS. She is a part time contact lens wearer. Binocularity and pupillary testing revealed no abnormality. A nterior segment ex am was remarkable for telangiectatic blood vessels on the palpebral conjunctiva superiorly and inferiorly $0 \mathrm{U}$ (those under the superior lid being more prominent). 0 ne area in particular OD looked like it had reoently bled (see frure 1). Fundoscopy showed healthy maculae and optic nerves with 0.25 aupping $0 U$. Peripheral fundosopy was performed undilated and revealed a large elevated retinal area superior nasal OD (see fgure 2). The patient was subsequently dilated to further ex amine the lesion. There was a deeper oolor to the lesion at the posterior border, though the central and anterior borders appeared slightly paler. The lesion was not visible on primary gaze.

\section{DISCUSSION}

The tentative diagnosis of the anterior segment finding was palpebral conjunctival telangiectasia causing hemorrhage, a finding consistent with and commonly found on mucous membranes in patients with hereditary hemorrhagic telangiectasia. For the potentially more concerning posterior segment finding, the tentative diagnosis of vortex vein varix was reached. Elevation of the vortex vein ampulla in peripheral gaze is a relatively common anatomic variation which is
Dr Russell Robertson OD 


\section{ARTICLE}

quite often overlooked or ignored. On occasion, this elevation can be very prominent to the point that it is notably out of the ordinary. In such cases, lesions known to elevate the retina must be ruled out, namely melanoma or other tumours, and other lesions such as choroidal neovascular membrane. It is in this differentiation that knowledge of the characteristics of vortex vein varices becomes important.

Literature describing varices of the vortex vein ampulla first appeared in 1967 when Rutnin described an elevation of some vortex vein ampullae toward the vitreous. He also noted that with pressure on the globe, he was able to flatten the lesions ${ }^{1}$. The term varix was first applied by O sher in $1981^{1}$. Many theories exist as to why theselesions occur, namely, extraocular muscle contraction, kinking of the vortex vein when the eye is rotated out of primary position, and simply a varicosity of a vein similar to those seen elsewhere in the body ${ }^{1}$. These theories are based on both what makes the lesions appear and disappear. Vortex vein varices are known to enlarge on peripheral gaze and disappear (or become much less prominent) on primary gaze. They are also known to flatten when pressure is applied to the globe. The elevation has also been elicited using the Valsalva maneuver and, as a result, the theories of extraocular muscle contraction and kinking of the vortex vein are believed to be somewhat less likely². Hereditary hemorrhagic telangiectasia is a condition in which varicosities are known to occur, raising the inference that the systemic condition may be related to the abnormally large vortex vein varix found in this patient's eye. Unfortunately, there is not a great deal of case related evidence in the literature to support this claim.

As mentioned earlier, the characteristics of these lesions become very important in distinguishing these lesions from other more sinister findings. The dynamic nature of the vortex vein varix is probably most important, in that the lesions flatten with pressure on the globe and on primary gaze. Hence, three mirror examination will not demonstrate the varix ${ }^{3}$. However, often a peripheral non-contact fundus lens examination will demonstrate the phenomenon quite nicely and in magnified views as compared to binocular indirect ophthalmoscopy. Vortex vein varices tend to be more often located nasally and can be 2-3 mm thick ${ }^{3,4}$. They may also show an increased visibility of the choroidal pigment at the posterior edge of the varix ${ }^{4}$. The lesion itself, though sometimes confused with other more serious abnormalities, is completely benign and asymptomatic.

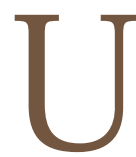
ne femme de 27 ans de race blanche s'est présentée à un ex amen avec des symptômes de saignement s'écoulant de l'œil. E lle n'avait pas de problèmes visuels, bien qu'elle ait remarqué une douleur au toucher sur sa paupière supérieure lorsqu'elle met une crtaine pression sur cette dernière. D e ses antéé dents médicaux, ainsi que des antécédents médicaux de sa famille, ressort principalement une télangiectasie hémorracique héréditaire. A u moment de l'ex amen, elle ne prenait aucun médicament.

L a patiente s'est présentée avec une acuité corrigée de 20/ 200 U. Son erreur de réfraction actuelle était de $-2,25-0,75$ x 002 OD et de $-3,25-0,50 \times 1800 \mathrm{~S}$. E lle porte des lentilles de contact à l'occasion. L'examen de la vision binoculaire et de la pupille n'a révéée aucune anomalie. D e l'examen du segment antérieur ressortent principalement des vaisseaux sanguins télangiectatiques sur la conjonctive palpébrale supérieure et inférieure $0 \mathrm{U}$ ( cux en dessous de la paupière supérieure étant plus importants). Il semblait qu'une région en particulier OD avait récemment saigné (fyure 1). L 'ophtalmosopie a révélé une macule en santé et des nerfs optiques avec une ex cavation de 0,25 0 U. L 'ophtalmosoopie périphérique a été effectuée sans dilatation et a révélé une région rétinienne élevée importante en nasal supérieur OD (fyure 2). On a par la suite dilatél'oil de la patiente afn d'ex aminer davantage la lésion. $0 \mathrm{n}$ a constaté une couleur plus intense à la limite postérieure de la lésion, bien que les bords ontraux et antérieurs apparaissaient légèrement plus pâles. L a lésion n'était pas visible en fix ation primaire.

\section{DISCUSSION}

Le premier diagnostic posé, selon les résultats sur le segment antérieur, était une télangiectasie conjonctive palpébrale causant des hémorragies, état que l'on constate couramment dans la muqueuse des patients qui souffrent de télangiectasie hémorragique héréditaire et qui correspond à ce diagnostic. Dans le cas du résultat possiblement plus inquiétant sur le segment postérieur, le premier diagnostic de varices de la veine vortiqueuse a été posé. L'élévation de l'ampoule de la veine vortiqueuse en fixation primaire est une variation anatomique plutôt courante qui est très souvent négligée ou ignorée. À l'occasion, cette élévation peut être très importante à un tel point qu'on la remarque facilement. Dans de tels cas, les lésions reconnues pour élever la rétine doi-

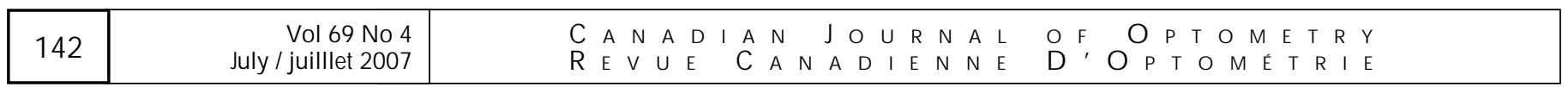




\section{ARTICLE ARTICLE}

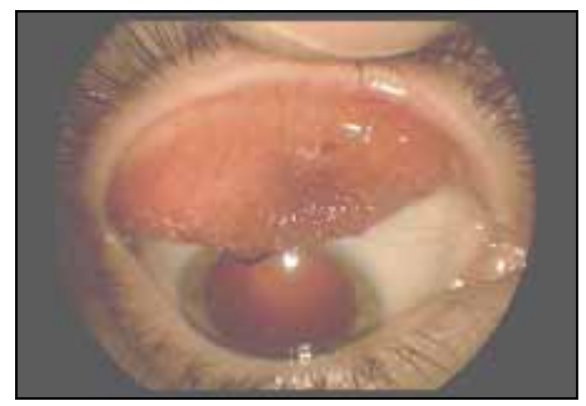

Figure 1 : palpebral conjunctival telangiectasia causing hemorrhage

Télangiectasie conjonctive palpébrale causant une hémorragie

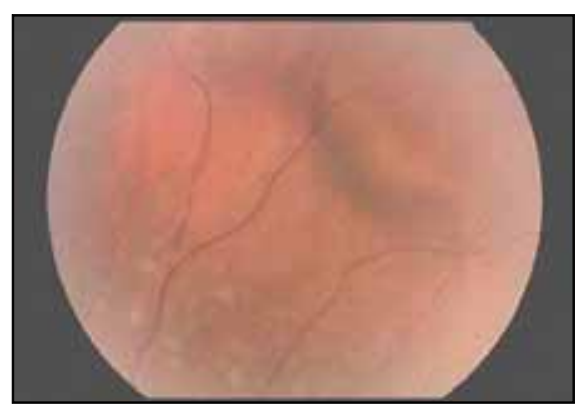

Figure 2 - vortex vein varix in patient with hereditary hemorrhagic telangiectasia

Varice de la veine vortiqueuse chez un patient souffrant de télangiectasie hémorragique héréditaire

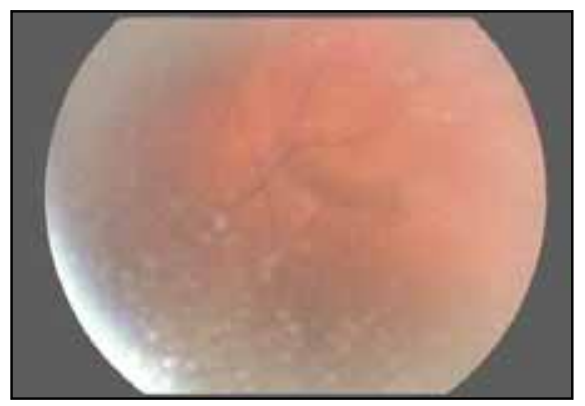

Figure 3 - vortex vein varix photo in another patient who does not have hereditary hemorrhagic telangiectasia

Photo d'une varice de la veine vorti-queuse chez un autre patient qui ne souffre pas de télangiectasie hémorragique héréditaire vent être exclues, notamment le mélanome ou autres tumeurs, ainsi que d'autres lésions comme la membrane néovasculaire choroïdienne. C'est dans cette différenciation que la connaissance des caractéristiques des varices de la veine vortiqueuse devient importante.

Les écrits décrivant les varices de l'ampoule de la veine vortiqueuse sont d'abord parus en 1967 lorsque Rutnin a présenté une description d'une élévation de certaines ampoules de la veine vortiqueuse vers le corps vitré. Il a également remarqué qu'en exerçant une pression sur le globe, il était en mesure d'aplatir les lésions1. Le terme varice a d'abord été appliqué par O sher en 1981'. Il existe de nombreuses théories sur la présence de ces lésions, notamment la contraction des muscles de l'orbite, la torsion de la veine vortiqueuse lorsque l'œil n'est pas dans sa position principale et tout simplementla varicosité d'une veine similaire à ce que l'on voit ailleurs dans le corps'. Ces théories sont basées sur ce qui fait apparaître et disparaître les lésions. Il est reconnu que les varices de la veine vortiqueuse prennent de l'expansion en fixation périphérique et disparaissent (ou deviennent beaucoup moins importantes) en fixation primaire. Il est également reconnu que lorsqu'on applique une pression sur le globe, elles s'aplatissent. L'élévation est également disparue à l'aide de la manœuvre de Valsalva et, par conséquent, on croit que les théories de la contraction des muscles de l'orbite et de la torsion de la veine vortiqueuse sont quelque peu moins probables². La télangiectasie hémorragique héréditaire est une affection caractérisée par l'apparition de varicosités. On doit donc en conclure que cette maladie systémique peut être reliée à la varice de la veine vortiqueuse de grosseur anormale que l'on a constatée dans l'œil de cette patiente. Malheureusement, on ne dispose pas de beaucoup de preuves découlant de cas dans les écrits pour appuyer cette allégation.

Comme il a été mentionné plus tôt, les caractéristiques de ces lésions deviennent très importantes pour distinguer ces lésions d'autres résultats plus sinistres. $\mathrm{La}$ nature dynamique de la varice de la veine vortiqueuse est probablementl'élémentleplusimportantétantdonnéque les lésions s'aplatissent lorsqu'une pression est exercée sur le globe et en fixation primaire. Par conséquent, l'examen à l'aide du verre à trois miroirs ne montrera pas la varice ${ }^{3}$. Toutefois, un examen périphérique sans contact du fond de l'œil à l'aide de lentilles montrera souvent très bien le phénomène et donnera une vue très agrandie de l'œil, comparativement à l'ophtalmoscopie indirecte binoculaire. Les varices de la veine vortiqueuse ont tendance à être plus souvent situées dans la région nasale et peuvent avoir une épaisseur de 2 à $3 \mathrm{~mm}^{3,4}$. Elles permettent également une plus grande visibilité du pigment choroïdien du côté postérieur de la varice4. La lésion elle-même, bien qu'elle soit parfois confondue avec d'autres anomalies plus graves, est complètement bénigne et ne provoque pas de symptômes.

\section{REFERENCES}

1. Lopez P. Varix of the vortex vein ampulla. J Am Optom Assoc. 1986 Feb; 57(2): 104-8.

2. O sher RH, Abrams GW, Yarian D, et al. Varix of the vortex ampulla. Am J O phthalmol. 1981 Nov; 92(5): 653-60.

3. Hunter JE. Vortex Vein Varix. Am J Optom Physiol Opt. 1983 Dec; 60(12): 995-6.

4. Levy J, Yagev R, Shelef I, et al. Varix of the vortex vein ampulla: a small case series. Eur J O phthal. 2005 May-Jun; 15(3): 424-7. 